\title{
HIGH-IONIZATION Fe K EMISSION FROM LUMINOUS INFRARED GALAXIES
}

\author{
K. Iwasawa ${ }^{1}$, D. B. Sanders ${ }^{2}$, A. S. Evans ${ }^{3}$, J. M. Mazzarella ${ }^{4}$, L. Armus ${ }^{5}$, and J. A. Surace ${ }^{5}$ \\ ${ }^{1}$ INAF-Ossservatorio Astronomico di Bologna, Via Ranzani, 1, 40127 Bologna, Italy \\ ${ }^{2}$ Institute for Astronomy, 2680 Woodlawn Drive, Honolulu, Hawaii 96822-1839, USA \\ ${ }^{3}$ Department of Astronomy, University of Virginia, 530 McCormick Road, Charlottesville, VA 22904 and NRAO, 520 Edgemont Road, \\ Charlottesville, VA 22903-2475, USA \\ ${ }^{4}$ IPAC, California Institute of Technology, Pasadena, CA 91125, USA \\ 5 Spitzer Science Center, California Institute of Technology, Pasadena, CA 91125, USA \\ Received 2008 December 24; accepted 2009 March 4; published 2009 March 24
}

\begin{abstract}
The Chandra component of the Great Observatories All-Sky Luminous Infrared Galaxy Survey (GOALS) presently contains 44 luminous and ultraluminous infrared galaxies (ULIRGs) with $\log \left(L_{\mathrm{IR}} / L_{\odot}\right)=11.73-12.57$. Omitting 15 obvious active galactic nuclei (AGNs), the other galaxies are, on average, underluminous in the 2-10 keV band by 0.7 dex at a given far-infrared luminosity, compared with nearby star-forming galaxies with lower star formation rates. The integrated spectrum of these hard X-ray quiet galaxies shows strong high-ionization $\mathrm{Fe} \mathrm{K}$ emission (Fe XXV at $6.7 \mathrm{keV}$ ), which is incompatible with X-ray binaries as its origin. The X-ray quietness and the Fe $\mathrm{K}$ feature could be explained by hot gas produced in a starburst, provided that the accompanying copious emission from high-mass X-ray binaries is somehow suppressed. Alternatively, these galaxies may contain deeply embedded supermassive black holes that power the bulk of their infrared luminosity and only faint photoionized gas is visible, as seen in some ULIRGs with a Compton-thick AGN.
\end{abstract}

Key words: galaxies: active - galaxies: starburst - infrared: galaxies - X-rays: galaxies

\section{INTRODUCTION}

While far-infrared (FIR) luminosity ${ }^{6}$ is often assumed to be a good indicator of the star formation rate (SFR) in extragalactic objects (e.g., Young \& Scoville 1991; Kennicutt 1998), it has also been argued that, in the absence of active galactic nuclei (AGNs), X-ray emission could trace the SFR as well; this latter assumption is based primarily on the observed correlation between X-ray luminosity (usually in the 2-10 keV energy range) and FIR luminosity initially found for nearby actively star-forming galaxies with SFRs 3-30 $M_{\odot} \mathrm{yr}^{-1}$ (e.g., Ranalli et al. 2003; Grimm et al. 2003; Gilfanov et al. 2004). This relation appears to extend to objects with higher SFR (up to $100 M_{\odot} \mathrm{yr}^{-1}$ ) and higher redshift (e.g., Hornschemeier et al. 2005; Persic \& Rephaeli 2007; Lehmer et al. 2008). The hard $\mathrm{X}$-ray emission is generally assumed to have a power-law form and to be predominantly due to the collective emission of highmass X-ray binaries (HMXBs), which are accreting compact objects (neutron stars or black holes) formed following the death of short-lived massive stars, and therefore expected to have a close relationship with the starburst.

There, however, have been indications that objects with very high computed SFRs, such as ultraluminous infrared galaxies (ULIRGs), might depart from the correlation because they are underluminous in X-rays for their computed SFRs (Persic \& Rephaeli 2007; Barger et al. 2007). Arp 220, the nearest ULIRG, is a prime example, with a $2-10 \mathrm{keV}$ luminosity $\sim 1$ dex below the general $L_{X}-L_{\text {FIR }}$ correlation (see Iwasawa et al. 2005). A further puzzle comes from its X-ray spectrum, which shows a strong high-ionization Fe $\mathrm{K}$ line (mainly Fe xxv, Iwasawa et al. 2005). This latter finding means that Arp 220 is not only Xray under-luminous, but that the hard X-ray emission is not primarily due to X-ray binaries because of the presence of a high-ionization Fe K line. In nearby star-forming galaxies,

\footnotetext{
$6 \quad L_{\text {FIR }} \equiv L(40-400 \mu \mathrm{m})$, as determined using the prescription described in
} Lonsdale et al. (1985)
Fe XXv is much weaker (e.g., Cappi et al 1999) or undetected, which is consistent that the hard X-ray emission is dominated by HMXBs. These peculiar properties of the nearest ULIRGs clearly warrant further investigation with a larger sample of (U)LIRGs.

The Great Observatory All-sky Luminous Infrared Galaxy Survey $(\text { GOALS })^{7}$ is a multiwavelength study of the most luminous infrared-selected galaxies in the local Universe, selected from the flux-limited IRAS Revised Bright Galaxy Sample (RBGS: Sanders et al. 2003). An overview of GOALS is given in Armus et al. (2009). C-GOALS (PI: D. B. Sanders) is the X-ray component of the project utilizing data from the Chandra X-ray Observatory (Chandra, hereafter). Details of the X-ray observations are described in K. Iwasawa et al. (2009, in preparation). Here we report results focusing primarily on the $L_{\mathrm{X}}-L_{\mathrm{FIR}}$ relation and the spectral properties of the hard X-ray emission, with special attention to the Fe K band.

The cosmology used to calculate luminosities in this paper is $H_{0}=70 \mathrm{~km} \mathrm{~s}^{-1} \mathrm{Mpc}^{-1}, \Omega_{\mathrm{M}}=0.3, \Omega_{\Lambda}=0.7$, based on the latest WMAP results (Hinshaw et al. 2009).

\section{THE SAMPLE}

The current C-GOALS sample is complete down to log $\left(L_{\mathrm{IR}} / L_{\odot}\right)=11.73$, and consists of 44 galaxies from the RBGS with redshifts $z=0.010-0.088$ (see Table 1). We first removed obvious AGN as follows. The primary criterion was a flat X-ray spectrum, assessed by the X-ray color or hardness ratio (HR). The $\mathrm{X}$-ray color is defined as $H R=$ $(H-S) /(H+S)$, where $H$ is the $2-8 \mathrm{keV}$ counts and $S$ is the $0.5-2 \mathrm{keV}$ counts. Objects with $H R>-0.3$ are classified as an AGN. This threshold is chosen because ULIRGs known to host AGNs (Mrk 231, Mrk 273, UGC 5101) cluster just above this value. All of the optically identified AGNs are

\footnotetext{
7 More information about GOALS is available at http://goals.ipac.caltech.edu/.
} 
Table 1

The C-GOALS Sample

\begin{tabular}{lrrlrr}
\hline \hline Object & $\begin{array}{c}\log L_{\mathrm{FIR}} \\
L_{\odot}\end{array}$ & $\begin{array}{r}\log L_{\mathrm{HX}} \\
\mathrm{erg} \mathrm{s}^{-1}\end{array}$ & Object & $\begin{array}{c}\log L_{\mathrm{FIR}} \\
L_{\odot}\end{array}$ & $\begin{array}{r}\log L_{\mathrm{HX}} \\
\mathrm{erg} \mathrm{s}^{-1}\end{array}$ \\
\hline HXQ sample & & & & & \\
F17207-0014 & 12.42 & 41.34 & NGC 3690 E & 11.49 & 41.00 \\
F19297-0406 & 12.39 & 41.26 & ESO 593-IG8 & 11.83 & 41.28 \\
P07251-0248 & 12.34 & $<40.90$ & VV 705 & 11.79 & 40.83 \\
F12112+0305 & 12.30 & 41.60 & ESO 255-IG7 & 11.75 & 41.46 \\
Arp 220 & 12.22 & 40.96 & F18293-3413 & 11.76 & 41.15 \\
F22491-1808 & 12.18 & 40.78 & F10173+0828 & 11.76 & 39.82 \\
F23365+3604 & 12.11 & 41.20 & ESO 203-IG1 & 11.84 & $<40.48$ \\
F10565+2448 & 11.99 & 41.20 & F01364-1042 & 11.82 & 41.18 \\
F15250+3608 & 11.98 & $<40.65$ & ESO 239-IG2 & 11.73 & 40.99 \\
F09111-1007 & 11.97 & 41.11 & P21101+5810 & 11.71 & 40.30 \\
ESO 286-IG19 & 11.98 & 41.32 & VV 250 & 11.68 & 41.49 \\
VII Zw31 & 11.89 & 41.43 & F10038-3338 & 11.69 & 40.80 \\
ESO 69-IG6 & 11.88 & 41.18 & ESO 77-IG14 & 11.67 & 41.15 \\
F17132+5313 & 11.84 & 40.93 & UGC 4881 & 11.65 & 40.73 \\
II Zw96 & 11.94 & 41.18 & IC 883 & 11.65 & 40.81 \\
AGN sample & & & & & \\
Mrk 231 & 12.37 & 42.48 & P19542+1110 & 12.06 & 42.61 \\
F14348-1447 & 12.35 & 41.77 & ESO 148-IG2 & 11.95 & 41.92 \\
P09022-3615 & 12.24 & 42.30 & UGC 5101 & 11.93 & 41.67 \\
P13120-5453 & 12.24 & 41.67 & NGC 3690 W & 11.32 & 41.00 \\
F14378-3651 & 12.16 & 41.53 & NGC 6240 & 11.81 & 42.54 \\
Mrk 273 & 12.15 & 42.40 & ESO 60-IG16 & 11.71 & 41.86 \\
F05189-2524 & 12.08 & 43.11 & VV 340a & 11.66 & 41.46 \\
F08572+3915 & 12.07 & 41.30 & & & \\
\hline & & & & &
\end{tabular}

Notes. Source names beginning with "F" or "P" are from the IRAS Faint Source Catalog or Point Source Catalog, respectively.

selected by this criterion. However, Compton-thick AGNs are generally missed by this criterion because of their weakness in the hard band. Therefore, objects that show a strong Fe K line at $6.4 \mathrm{keV}$, a characteristic signature of a Compton-thick AGN, are also classified as AGNs (NGC 6240, NGC 3690 West, VV 340a). These criteria classify 15 objects as AGN, and they are excluded from further discussion leaving an "hard X-ray quiet" (HXQ, as defined by their small HR) sample of 30 objects (including NGC 3690 East). The SFR of these 30 HXQ galaxies, calculated assuming that their FIR luminosity is due to dust heated by star formation alone, ranges from 60 to $300 M_{\odot} \mathrm{yr}^{-1}$.

\section{L(2-10 keV) AND L(FIR) RELATION}

The sensitivity of Chandra declines steeply above $7 \mathrm{keV}$. The $2-10 \mathrm{keV}$ luminosity is estimated by extrapolating the spectral model that describes the data up to $7 \mathrm{keV}$. When multiple hard $\mathrm{X}$-ray sources are present in a single object, those which have no Spitzer-MIPS $24 \mu \mathrm{m}$ counterpart are excluded for calculating the $2-10 \mathrm{keV}$ luminosity, assuming they have no relation with the IRAS measured luminosity. In NGC 3690, the western and eastern galaxies are treated separately due to the difference in classification, and their FIR luminosity ratio is assumed to be 1:2 based on the $38 \mu \mathrm{m}$ study (Charmandaris et al. 2002). The $\mathrm{X}$-ray luminosity is as observed in the rest-frame $2-10 \mathrm{keV}$ band, corrected only for Galactic absorption. The median value of the $2-10 \mathrm{keV}$ luminosity is $1.4 \times 10^{41} \mathrm{erg} \mathrm{s}^{-1}$ (which also matches the mean).

Figure 1 shows a plot of hard X-ray luminosity, $L(2$ $10 \mathrm{keV})$, versus FIR luminosity, $L(40-400 \mu \mathrm{m})$, for our complete sample of 44 RBGS galaxies listed in Table 1. For

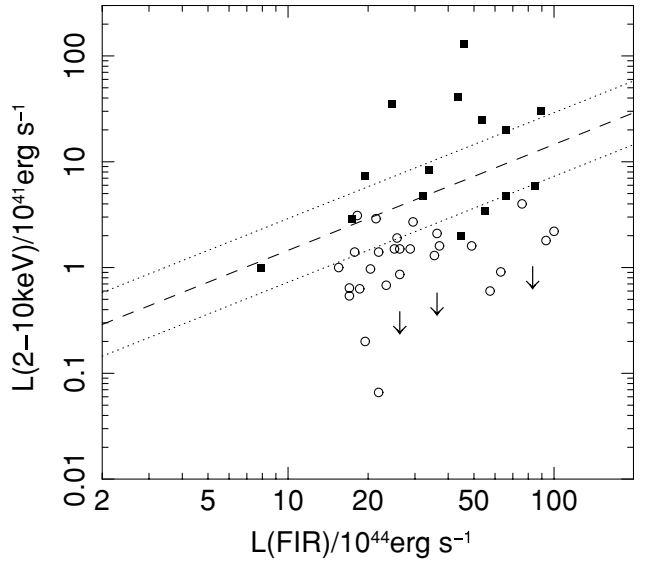

Figure 1. $L(2-10 \mathrm{keV})$ vs. $L(\mathrm{FIR})$ for the GOALS AGN (filled squares) and HXQ (open circles) galaxies listed in Table 1. For the three objects with no detection in the $2-10 \mathrm{keV}$ band, the $95 \%$ upper limits derived by following Kraft et al. (1991) are shown. The dashed line indicates the correlation for the sample of Ranalli et al. (2003). The dotted lines show a factor of 2 above and below the Ranalli et al. correlation, within which the other studies of the same relationship lie.

the GOALS HXQ galaxies alone, no clear correlation is seen (Kendal's $\tau \simeq 0.3$ ) in the limited FIR luminosity range. The correlation obtained by Ranalli et al. (2003) is rederived in the same way as done for our sample: $\log L(2-10 \mathrm{keV})=-3.84$ $\log L$ (FIR). This is fully consistent with the original relation with an offset due to the wider FIR wavelength range. The $L_{X}-$ SFR correlations studied by various authors (e.g., Grimm et al. 2003; Persic \& Rephaeli 2007; Lehmer et al. 2008) all lie within a factor of 2 of Ranalli et al. (2003) as indicated in the figure. The correlation line is consistent with the upper envelope of the HXQ sample. Most of our HXQ galaxies lie well below this correlation. The median value of the logarithmic ratio of $L(2-$ $10 \mathrm{keV}$ ) and $L(\mathrm{FIR})$ is -4.5 , which means our sample of HXQ galaxies is $\sim 0.7$ dex underluminous in the $2-10 \mathrm{keV}$ band for a given $L(F I R)$, or corresponding SFR. We note that, given the lack of correlation in our sample, this is merely a comparison of the average value relative to the $L_{\mathrm{X}}-L_{\mathrm{FIR}}$ correlation.

\section{THE INTEGRATED 4-8 keV SPECTRUM}

We investigate the integrated hard band spectrum of the $30 \mathrm{HXQ}$ galaxies, since they are too faint in hard X-rays to allow an individual inspection for the iron line. Although detection of the Fe $\mathrm{K}$ line has been reported for the XMM-Newton spectra of NGC 3690 East (Ballo et al. 2004) and Arp 220 (Iwasawa et al. 2005), the line does not have sufficient counts in the Chandra data for a significant detection (see e.g., Clements et al. 2002). These two objects are included in the integrated hard-band spectrum and their contribution to the total spectrum is also investigated. In cases where multiple hard X-ray sources are detected in a single object (IRAS F09111-1007, ESO 255IG007, VV 250, ESO 77-IG014, IRAS F12112+0305, VV 705, UGC 4881), multiple apertures were selected accordingly.

Given that our primary interest is in the $\mathrm{Fe} \mathrm{K}$ properties, we restrict the analysis to the $\mathrm{Fe} \mathrm{K}$ and neighboring band (4.25-7.65 keV in the rest frame). The Fe K complex generally consists of two components with distinct ionization states: (1) cold $\mathrm{Fe}$ at $6.4 \mathrm{keV}$; and (2) highly ionized $\mathrm{Fe} \mathrm{xxv}(6.7$ $\mathrm{keV})$ with a minor contribution from Fe XxVI $(7.0 \mathrm{keV})$. These two components can be separated well at the spectral resolution of the Chandra ACIS-S. We define two spectral bands centered on the respective line components $(6.35-6.55 \mathrm{keV}$ and 


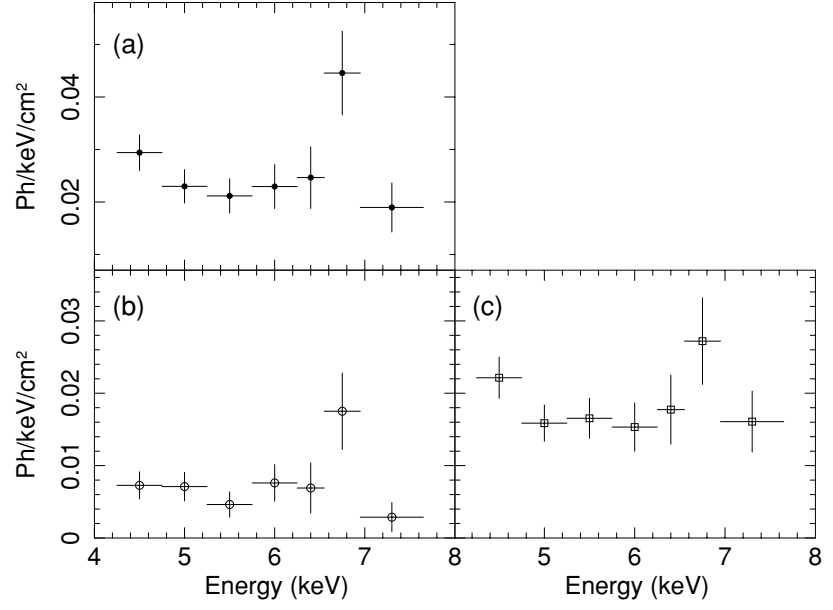

Figure 2. (a) The integrated 4.25-7.65 keV spectrum of the 30 HXQ objects in Table 1. The energy is in the rest frame. An excess in the high-ionization Fe $\mathrm{K}$ band (primarily due to Fe Xxv) at $6.55-6.95 \mathrm{keV}$ is clearly visible. The continuum is flat $(\Gamma=1.1 \pm 0.5)$ and the $\mathrm{Fe} \mathrm{K}$ band excess has $E W \sim 1 \mathrm{keV}$. (b) The integrated spectrum of Arp 220 and NGC 3690 E, two Fe XXV emitters known from XMM-Newton observations. (c) The integrated spectrum of the HXQ sample excluding Arp 220 and NGC 3690 E. Even without the two known Fe Xxv emitters, a comparable excess flux above the continuum in the Fe XXv band is present, indicating that other galaxies make a significant contribution to the total line flux in a).

6.55-6.95 keV), and five neighboring bands for the continuum, giving seven spectral bands over the range $4.25-7.65 \mathrm{keV}$ in the rest frame.

The hard X-ray sources of our sample are generally compact, and a small extraction radius, typically $\sim 1^{\prime \prime} 5-2^{\prime \prime}$, is used. The detected counts have been corrected for background using the data from a source-free area on the detector in the same observation, although the correction is almost negligible with such a small aperture.

For individual objects, source counts recorded in seven bands corresponding to the pre-defined rest-frame bands were accumulated. The detector response curve was corrected by dividing by the mean effective area of each spectral band. As most sources have only a few counts over the energy range, stacking was done as a straight integration of individual sources, neither normalizing by the exposure time nor source brightness.

The total source counts accumulated in the rest-frame 4.25$7.65 \mathrm{keV}$ band for the HXQ sample are 296 counts. The integrated spectrum is shown in Figure 2(a). A strong Fe K line is immediately recognized with a $3 \sigma$ excess. The line peaks at the high-ionization Fe $\mathrm{K}$ band $(6.55-6.95 \mathrm{keV})$, indicating that the line is primarily due to Fe xxv from a highly ionized medium. The total counts collected in this line band are 35 counts. There might also be a slight excess in the $6.4 \mathrm{keV}$ line band, which is however not significant.

Given the limited statistics, a further analysis beyond the line detection is not warranted. However, given our stacking method, which is biased for sources that are bright and/or with a long exposure, it is at least worth examining whether Arp 220 and NGC $3690 \mathrm{E}$, the two known Fe K emitters from their XMMNewton spectra, dominate the line detection. These two objects and the rest of the HXQ sample were integrated separately, and their spectra are presented in Figures 2(b) and (c). Both spectra show $\mathrm{a} \simeq 2 \sigma$ excess in the Fe Xxv band with comparable line fluxes. This means that, besides Arp 220 and NGC 3690 $\mathrm{E}$, there is a significant contribution by other galaxies to the detected line flux. The higher continuum level in the spectrum of the sample without Arp 220 and NGC 3690 E (Figure 2(c)) suggests that some sources without strong $\mathrm{Fe} \mathrm{K}$ emission are present, and that they may be dominated by HMXBs as found in nearby star-forming galaxies.

Fitting a power law to the continuum gives a photon index of $\Gamma=1.1 \pm 0.5$, indicating a rather hard continuum above $4 \mathrm{keV}$. The equivalent width of the excess in the $\mathrm{Fe} \mathrm{K}$ band with respect to the power-law continuum is $E W=0.9 \pm 0.3 \mathrm{keV}$. The $90 \%$ upper limit of the EW for a $6.4 \mathrm{keV}$ line is $120 \mathrm{eV}$.

Fitting a thermal spectrum (MEKAL) gives a temperature of $k T=8 \pm 2 \mathrm{keV}$ and an Fe metallicity consistent with the solar value. Considering that the integrated spectrum is diluted by some sources that contribute only to the continuum emission (see above), the Fe metallicity of the line-emitting sources is likely to be supersolar.

Regardless of the choice of spectral model, the strong highionization $\mathrm{Fe} \mathrm{K}$ line is not compatible with the $\mathrm{X}$-ray spectrum of known HMXBs. Fe K emission is often observed from HMXBs in our Galaxy, but the major component is the cold line at $6.4 \mathrm{keV}$ and its EW is on average $\sim 200-300 \mathrm{eV}$ (e.g., White et al. 1983). The much stronger, high-ionization line in the integrated spectrum of the HXQ sample suggests that X-ray binaries are not the primary source of the $4-8 \mathrm{keV}$ emission.

\section{DISCUSSION}

The general quietness of hard X-ray emission in the HXQ galaxies (Figure 1) is likely related to the detection of the highionization Fe $\mathrm{K}$ feature, which rules out $\mathrm{HMXBs}$ as the primary origin of the faint hard $\mathrm{X}$-ray emission.

The powerful FIR emission in the HXQ (U)LIRGs predicts an abundance of HMXBs if star formation is the dominant power source. The Chandra spectra, however, suggests these sources contribute little to the hard X-ray emission. Therefore, either the bulk of the HMXBs is missing or they are heavily obscured from view. Suppose heavy obscuration is the cause. Since the Fe K band is still transparent to $N_{\mathrm{H}} \sim 10^{24} \mathrm{~cm}^{-2}$, the obscuring column has to be larger, perhaps of the order of $10^{25} \mathrm{~cm}^{-2}$. A dense, nuclear molecular disk (with a dynamical mass of the order of $10^{9} M_{\odot}$ ) is often found in the central part of ULIRGs (e.g., Scoville et al. 1997; Bryant \& Scoville 1999) and thus, such a large column may not be unrealistic. The size of the nuclear molecular disk is generally found to be a few $100 \mathrm{pc}$ but the densest part can be as small as $30 \mathrm{pc}$, as found by high-resolution studies of Arp 220 (Downes \& Eckart 2007; Sakamoto et al. 2008; Aalto et al. 2008), where a rough estimate of the density is $10^{5}-10^{6} \mathrm{~cm}^{-3}$. According to Grimm et al. (2003), the number of HMXBs with $L_{X}>2 \times 10^{38} \mathrm{erg} \mathrm{s}^{-1}$, which would dominate the integrated luminosity of the entire HMXB population, is $\sim 300\left(\mathrm{SFR} / 100 M_{\odot} \mathrm{yr}^{-1}\right)$. For SFR $=$ $200 M_{\odot} \mathrm{yr}^{-1}$, as estimated for Arp 220, the number is $\sim 600$, and most of them need to be confined within $30 \mathrm{pc}$ in order for their radiation to be suppressed. The required stellar density is unusually high and could be a problem in this particular case. The other galaxies may have a larger starburst region and could avoid this problem provided the required mass of the obscuring gas does not exceed the dynamical mass.

Once the HMXBs are obscured from view, the Fe XXV spectrum can be explained by high-temperature $\left(T \sim 10^{8} \mathrm{~K}\right)$, thermal gas produced by a starburst. Possible sources are (1) an internally shocked hot bubble ${ }^{8}$ produced by thermalizing the

\footnotetext{
8 This should be distinguished from a much more extended, soft X-ray nebula, which most likely originate from the swept-up interstellar medium (e.g., Tomisaka \& Ikeuchi 1988).
} 
energy of supernovae ( $\mathrm{SNe}$ ) and stellar winds, as predicted by, e.g., Chevalier \& Clegg (1985); and (2) collective luminous $\mathrm{SNe}$. The latter is ruled out if HMXBs are all embedded in heavy obscuration because the $\mathrm{SNe}$ should also be embedded in the same obscuring material. The former would excavate the obscuration and become visible. With the assumed high SFR, the luminosity and the spectrum with strong Fe XXV can be reproduced (e.g., Iwasawa et al. 2005 for Arp 220). The diffuse hard X-ray emission seen in M 82 (Griffiths et al. 2000; Strickland \& Heckman 2007; Ranalli et al. 2008), after removing resolved X-ray binaries, has, in fact, a comparable X-ray to FIR ratio with that of our GOALS HXQ sample. The strong Fe line is also well matched with the prediction for metal enriched gas of this origin.

With the presence of dense nuclear gas, a heavily obscured AGN is also a possible explanation for the X-ray spectra of our HXQ sample. Massive black holes in the process of rapid growth are naturally expected to be present in (U)LIRGs. When a central AGN is deeply buried with a covering factor nearly unity, reprocessed light from the obscuring matter would have difficulty escaping. The high-ionization Fe K line could then originate from extended, low density gas which is photoionized by the AGN. In a number of Compton thick Seyfert 2 galaxies, e.g., NGC 1068, Fe XXv has been seen (but normally weaker than the cold line at $6.4 \mathrm{keV}$ ). This is in fact the feature originally predicted by Krolik \& Kallman (1987) when assuming a likely scattering medium to produce the polarized broad-line region in NGC 1068 (Antonucci \& Miller 1985). Where the cold X-ray reflection is suppressed, the weak, highly photoionized gas could be the only observable feature. We note that Fe XXV has been observed as the primary Fe $\mathrm{K}$ feature in some Compton-thick AGNs residing in the ULIRGs IRAS F00183-7111 (Nandra \& Iwasawa 2007; Ruiz et al. 2007), the Super-Antennae (V. Braito et al. 2009), and possibly UGC 5101.

In Arp 220, recent measurements of a compact dust emission source with a steep temperature gradient in the western nucleus provides an argument for an AGN (Downes \& Eckart 2007; Aalto et al. 2008; but see Sakamoto et al. 2008). Millimeter wavelength molecular line observations also suggest the presence of X-ray-dominated chemistry, which favors heating by AGNs (Aalto et al. 2007; Imanishi et al. 2007; e.g., Meijerink \& Spaans 2005 for theory). The similarity of the X-ray properties suggests that the same explanation might apply for at least some of the GOALS HXQ galaxies-i.e., despite the lack of outward evidence of an AGN, a significant fraction of the infrared output could be powered by a heavily obscured AGN.
This research was supported in part by NASA through Chandra award Number G07-8108A, issued by the Chandra $X$-Ray Observatory, which is operated by the Smithsonian Astrophysical Observatory for and on behalf of NASA under contract NAS8-39073. We acknowledge use of the NASA/IPAC Extragalactic Database (NED), and the software packages CIAO and HEASoft.

\section{REFERENCES}

Aalto, S., Wilner, D., Spaans, M., Wiedner, M. C., Sakamoto, K., Black, J. H., \& Caldas, M. 2008, A\&A, 493, 481

Antonucci, R. R. J., \& Miller, J. S. 1985, ApJ, 297, 621

Armus, L., et al. 2009, PASP, submitted

Ballo, L., Braito, V., Della Ceca, R., Maraschi, L., Tavecchio, F., \& Dadina, M. 2004, ApJ, 600, 634

Barger, A. J., Cowie, L. L., \& Wang, W.-H. 2007, ApJ, 654, 764

Braito, V., et al. 2009, A\&A, submitted

Bryant, P. M., \& Scoville, N. Z. 1999, AJ, 117, 2632

Cappi, M., et al. 1999, A\&A, 350, 777

Charmandaris, V., Stacey, G. J., \& Gull, G. 2002, ApJ, 571, 282

Chevalier, R. A., \& Clegg, A. W. 1985, Nature, 317, 44

Clements, D. L., McDowell, J. C., Shaked, S., Baker, A. C., Borne, K., Colina, L., Lamb, S. A., \& Mundell, C. 2002, ApJ, 581, 974

Downes, D., \& Eckart, A. 2007, A\&A, 468, L57

Gilfanov, M., Grimm, H.-J., \& Sunyaev, R. 2004, MNRAS, 347, L57

Griffiths, R. E., Ptak, A., Feigelson, E. D., Garmire, G., Townsley, L., Brandt, W. N., Sambruna, R., \& Bregman, J. N. 2000, Science, 290, 1325

Grimm, H.-J., Gilfanov, M., \& Sunyaev, R. 2003, MNRAS, 339, 793

Hinshaw, G., et al. 2009, ApJS, 180, 225

Hornschemeier, A. E., Heckman, T. M., Ptak, A. F., Tremonti, C. A., \& Colbert, E. J. M. 2005, AJ, 129, 86

Imanishi, M., Nakanishi, K., Tamura, Y., Oi, N., \& Kohno, K. 2007, AJ, 134 2366

Iwasawa, K., Sanders, D. B., Evans, A. S., Trentham, N., Miniutti, G., \& Spoon, H. W. W. 2005, MNRAS, 357, 565

Kennicutt, R. C., Jr. 1998, ARA\&A, 36, 189

Kraft, R. P., Burrows, D. N., \& Nousek, J. A. 1991, ApJ, 374, 344

Krolik, J. H., \& Kallman, T. R. 1987, ApJ, 320, L5

Lehmer, B. D., et al. 2008, ApJ, 681, 1163

Lonsdale, C. J., Helou, G., Good, J. C., \& Rice, W. L. 1985, Cataloged Galaxies and Quasars Detected in the IRAS Survey (JPL D-1932) (Pasadena, CA: JPL)

Meijerink, R., \& Spaans, M. 2005, A\&A, 436, 397

Nandra, K., \& Iwasawa, K. 2007, MNRAS, 382, L1

Persic, M., \& Rephaeli, Y. 2007, A\&A, 463, 481

Ranalli, P., Comastri, A., Origlia, L., \& Maiolino, R. 2008, MNRAS, 386, 1464

Ranalli, P., Comastri, A., \& Setti, G. 2003, A\&A, 399, 39

Ruiz, A., Carrera, F. J., \& Panessa, F. 2007, A\&A, 471, 775

Sakamoto, K., et al. 2008, ApJ, 684, 957

Sanders, D. B., Mazzarella, J. M., Kim, D.-C., Surace, J. A., \& Soifer, B. T. 2003, AJ, 126, 1607

Scoville, N. Z., Yun, M. S., \& Bryant, P. M. 1997, ApJ, 484, 702

Strickland, D. K., \& Heckman, T. M. 2007, ApJ, 658, 258

Tomisaka, K., \& Ikeuchi, S. 1988, ApJ, 330, 695

White, N. E., Swank, J. H., \& Holt, S. S. 1983, ApJ, 270, 711

Young, J. S., \& Scoville, N. Z. 1991, ARA\&A, 29, 581 